\title{
Chapter 12: Building trust and companionship in e-coaching through embodiment
}

\author{
Mira El Kamali ${ }^{1}$, Leonardo Angelini ${ }^{1}$, Maurizio Caon ${ }^{1,2}$, Giuseppe Andreoni, \\ Nick Dulake $^{4}$, Paul Chamberlain ${ }^{4}$, Omar Abou Khaled ${ }^{1}$, Elena Mugellini' ${ }^{1}$ \\ ${ }^{1}$ University of Applied Western Switzerland (HES-SO), Humantech institute, 1700 Fribourg \\ ${ }^{2}$ School of Management, Fribourg, Switzerland \\ ${ }^{3}$ University of Politecnico di Milano, Dip. di Design, \\ ${ }^{4}$ Lab4Living, Sheffield Hallam, University, UK
}

\begin{abstract}
E-coaches are promising tools for health interventions, being scalable and relatively low costs compared to human coaches. Nevertheless, they cannot compare to humans when it comes to trust and social companionship. Hence, many researches were held around the importance of tangible user interfaces in building trust and companionship during human-machine interaction. Co-design, in particular, is the act of creating with stakeholders within the design development process to ensure the results meet their needs and are usable. This chapter describes a design process method and the rationale for building an embodiment for a companion and a trustable e-coach. We also shed lights on the co-design process held in the NESTORE project, which was specifically conducted with older adults to build a vocal assistant that supports user's behavioral change, as well as to increase the social bonding with the NESTORE e-coach.
\end{abstract}

\section{1 Introduction}

At the beginning of the 21 st century, researchers in Human-Computer Interaction started to theorize and implement interfaces that where bridging the physical and digital worlds, using representations and tools belonging the physical world to interact with the digital realm. Indeed, Tangible User Interfaces [1] aimed at establishing a direct coupling between digital information and physical objects, these latter acting as both representation and control for information that was traditionally constrained behind a screen. Few examples of tangible interaction exist in commercial products, this is mainly due to the challenges related to production and maintenance of physical interfaces. As screen-based interfaces do not have physical parts that can break and can be easily updated at software level, they are generally preferred as main interfaces for connected objects [2]. However, some research studies 
demonstrated the benefits of tangible user interfaces for older adults, thanks to their intuitiveness and fast learning curve [3]. Bong et al[4] found 21 studies using tangible user interfaces for supporting social interactions in older age. In particular, they highlighted that many studies lacked user involvement and of interdisciplinary collaborations during the design process. Moreover, authors reported that many studies did not test the prototypes presented in the article. In opposition, Co-design is a core method of approach adopted and embedded by the NESTORE consortium [5] in the research and development of the NESTORE system. We have developed co-design activity adopting co-design methods and tools. Through workshops, the experts in co-designing in this project have developed further tools to facilitate codesign within the workshops they have conducted with the Expert by experience group (UK), as well as to support non-design NESTORE project partners in the cocreation and development of NESTORE.

This chapter focuses on the design process to create a tangible interface, more specifically for creating an embodied conversational agent that able to build trust and companionship with the user. Section 2 presents a theoretical background of tangible embodiments conversational agents. Section 3 presents a design process method, whereas, section 4 describes a use-case for the aforementioned co-design method: the NESTORE project case and its three design concepts derived from user requirements. Finally, Section 5 presents the final design of the NESTORE tangible coach.

\subsection{Background on tangible conversational agents in increas- ing trust and companionship}

Conversational agents might be presented to the user in different forms. In the last years, the most common forms of interaction modality with conversational agents are chats (i.e., chatbots available in messaging applications such as Facebook Messenger and Telegram) and vocal assistants (e.g., Apple Siri, Google Assistant, Microsoft Cortana). In both cases, the users have a limited understanding of the physical form of the agent. In the first case, this might be limited to the chatbot avatar, and in the second case only to the voice gender and pitch. Having a full embodied conversational agent might help the user to increase the trust on the agent and to build a stronger emotional bonding. At the same time, the conversational agent embodiment is important to set user expectations in terms of conversation abilities. While users would be happy to discuss and interact with human-like agents, the technology is very often not ready to meet these expectations. In this case, when the agent embodiment is too close to humans, but its behavior or form is not close enough to be perceived by the user as such, the user often develops a feeling of rejection, also known as the "Uncanny Valley" phenomenon [6]. El Kamali et al [7] conducted a systematic literature review that aimed at individuating previous ecoaching interventions for promoting healthy lifestyles in older adults [ref]. As part of it, they analyzed the different types of e-coach embodiments used so far and the 
insights found in previous studies, concerning different design choices. They found that not all e-coaches had a proper embodiment (in some cases only a simple digital intervention was implemented) and most embodiments were purely virtual representation of the e-coach, with no physical representation of the e-coach. In the next section, we delve more into the embodiments designed for the e-coaches for promoting healthy lifestyles in older adults.

\section{3 Design Process Method}

This section of the chapter focuses on a co-design process method. Co-design is the act of creating the design with users during the development. This will make sure that the results meet user's needs. The design process described by Chamberlain et al [8], grounds on an iterative co-design. Error! Reference source not found. resumes the different steps of the method described in [8] of the co-design process method. Workshops can be carried out with the "exhibition in the box" methodology which allows to determine general requirements for technological devices and most of them can be applied also to design the main product. Conducting the same type of workshop in different countries cab be helpful to derive specific requirements for the design from different sociocultural point of views. In particular, in the case of NESTORE tangible coach project, 4 countries were concerned: Italy, Spain, UK, The Netherlands. The workshop in UK focused on the co-design of the tangible coach, while interesting inputs and suggestions about the tangible coach appearance, personality and interaction modalities were discussed also in the other workshops (in Spain, The Netherlands and Italy).

As a next step, based on the requirements and the ideas produced by users during the workshops, different complementary designs are conceived, each answering different user needs. The different designs are then shown to the users in the next weeks to collect feedback about each solution, allowing them to build on the different design concepts and finally to propose new ideas, inspired by elements from each design. Finally, the analysis of such feedback can be used to understand the general user preferences and drives, to be integrated in the final design. The final design should be then prototyped and tested with the target users. 


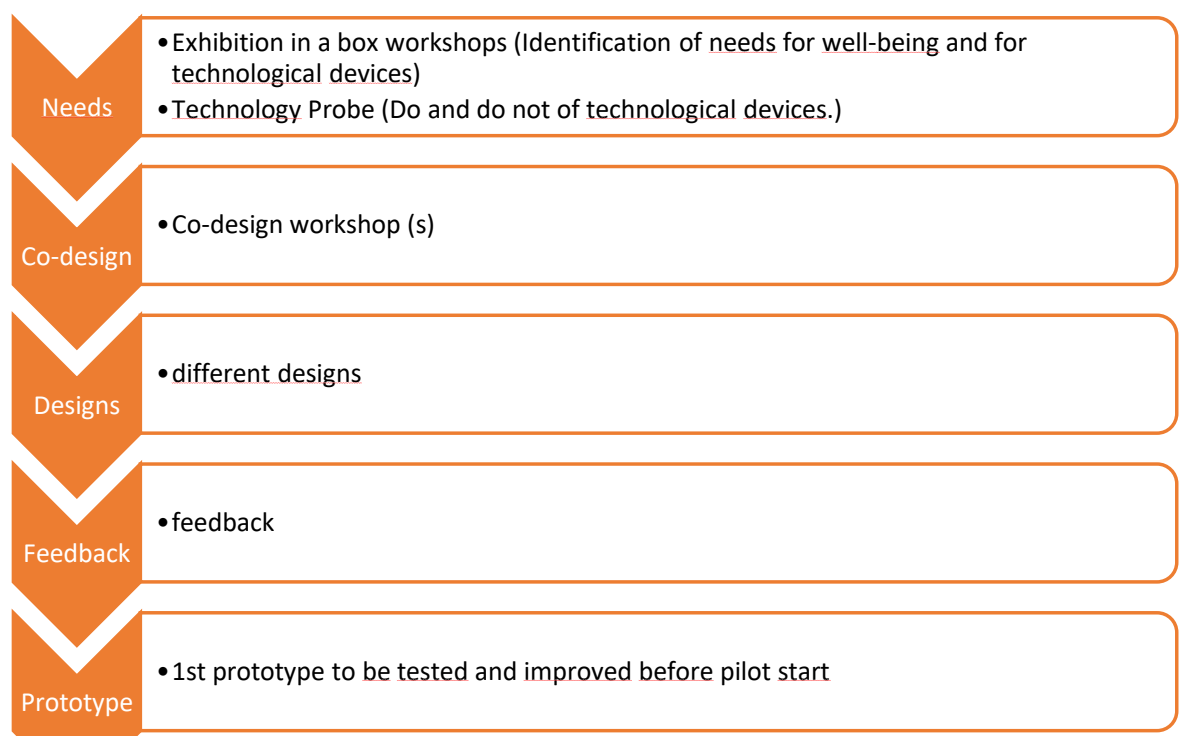

Fig. 12.1: Co- Design Process Example

\section{4 NESTORE tangible coach co-design}

NESTORE is a virtual coach that aims to help, improve and maintain the wellbeing of older adults. This virtual coach comes in different forms, distributed across different devices. One of the forms of the NESTORE coach is a conversational agent. The conversational interfaces are textual, through a chatbot embodied in a mobile application, and vocal through an embodiment in a physical device. The main goal of these type of interfaces is to have a natural conversation with the user. This helps to create a sense of companionship and bond through communication. Tangible interfaces, on the other hand, might be beneficial for supporting an emotional bonding with the e-coach embodiments and for stimulating self-reflection on personal wellbeing and for sustaining motivation towards the goal. 


\subsubsection{Preliminary Designs}

We carried out a number of activities in order to identify user needs. As a result, below is a list of user requirements based on the results from the NESTORE tangible coach co-design workshops in Spain, Italy, UK and the Netherlands.

\section{R1. Intelligible/controllable \\ R2. Robust \\ R4. Affordable \\ R5. Personalized \\ R6. Easy-to-use/learn \\ R7. Easy Authentication \\ R8. Accessible for all \\ R9. Transportable \\ R10. Appearance \\ R11. Context-Aware \\ R12. Unobtrusive \\ R13. Friendly}

Three different designs were derived then from user requirements and from previous findings found during the literature analysis.

\subsubsection{Familiar Coach}

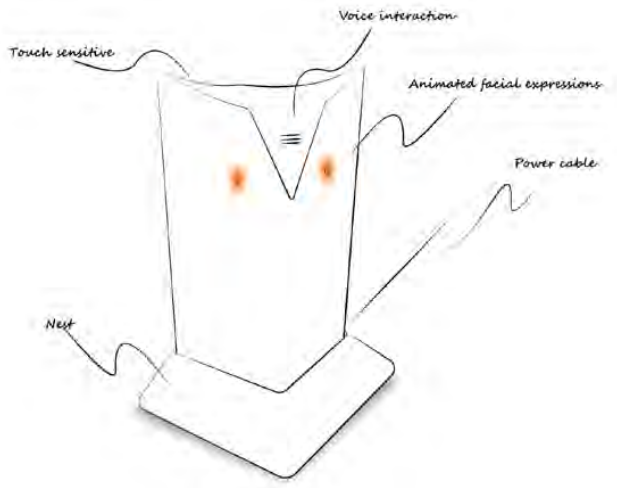

Fig. 12.2: Co- Design Concept of the familiar coach

The appearance of the familiar Coach resembles a knowledgeable bird and has design characteristics that are understated but recognisable (R10). The Familiar Coach 
is portable and can be moved around the house (R9). It has Nests setup in different rooms and can be easily moved from one Nest to another (R3). It is aware of its location and changes it coaching attitude to suit that location (R11), E.g. peaceful in the bedroom, reduced notifications (R12). The coaching attitude in locations can be personalised through the NESTORE App (R5). It uses Voice interaction (R6, R8) as its primary mode of communication supported by animated facial expressions on the exterior surfaces of the design as an indication of progress, notifications and a need that it would like to communicate with you. Areas of the Familiar Coach are touch sensitive, these areas are used to invoke communication, so you are in control (R1, R12). Users can have a conversation on a new coaching activity or to ask for progress/help on a current activity. If there is a lot of information to be passed on by the Familiar Coach, it will send useful tips and suggestions via text or email.

\subsubsection{Growing Coach}

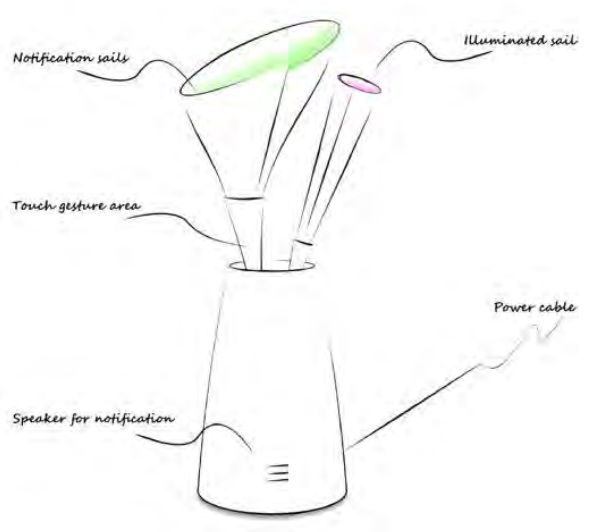

Fig. 12.3: Co- Design Concept of the Growing coach

The Growing Coach would resemble a collection of elegant stems (R10). At the top of the stems are notification sails that unfurl to show progress and achievements throughout coaching activities (R12). The group of notification sails will show overall progress with slow movement and other sails will move quickly to show task achievement. The Growing Coach is designed to be in a fixed social space in the house (R3) and used as a touchpoint throughout the day (R7). It always shows progress notifications, but will wait for your presence to show achievements (R11). It will start by getting your attention using a soft sound before starting the notification sail movement. At the center of the notification sail there is a light source which illuminates the sail with different colors to indicate the NESTORE coaching activity. This can be personalized through the NESTORE APP to aid recognition (R5). With a touch gesture on the stem, the Growing Coach would repeat the last 
achievement notification (R6, R8). You can communicate with the Growing Coach via the NESTORE App and Chatbot.

\subsubsection{Compact Coach}

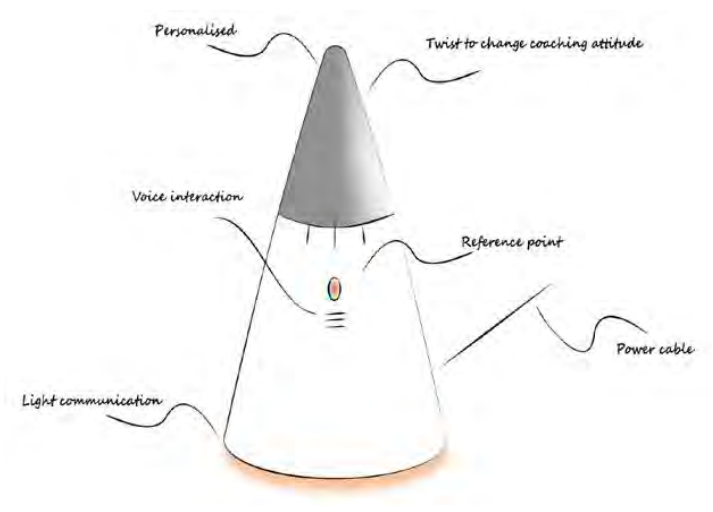

Fig. 12.4: Co- Design Concept of the compact coach

The compact vocal coach is of stylish, solid geometric design that blends in with your environment (R10). Elements can be personalised (R5), such as the ability to the outer surface of the product and the wake word that you use to start a conversation can be changed (R8). It uses light communication to gain your attention when it wants to give you an update on your progress (R12). The compact vocal coach would have a light/group of lights at the front as a reference point, so that you know that you are speaking in the correct direction and that the coach is listening (R6). The attitude of the coach can be changed through tangible manipulation of the design and will give a visual reference on the outer surface of the compact coach to show the current coaching attitude (R1). Like a friend (r13), the compact vocal coach will send you a text from time to time, with some tips or suggestions that are relevant to your activities. This might be a link to a YouTube video on cooking. The Compact Vocal Coach contains a battery, which means (if needed) it could be moved around the house for periods of time (R9) without having to find a plug (R3).

\subsubsection{Survey and user feedback}

We sent a survey for 7 older people from the UK, 6 from Spain, 10 from the Netherlands and 10 participants from Italy, who preferred the Compact Coach design and voice interaction, visual cues, ease of charging(portability), appearance and ease of control were the most positively perceived.

\subsubsection{Tangible coach final prototype:}


The final design of the Tangible Coach is oval in shape, is white, and made of $3 \mathrm{D}$ printed plastic with a textile cover for a warm and emotional feel to the touch, meant to encourage tangible handling. The aim was to create a feeling of camaraderie. The tangible coach has its main face which is when the device is positioned on its side. This oval face is the main part of this body which looks like a human face. Users can communicate with it through its different lighting models. His human face expresses a specific act. Such acts are "Listening state" or "Thinking state" or "Speaking state" (Figure 3). These acts are represented by LED patterns. The tangible coach has two main positions: Sleep state, where the tangible coach cannot hear or interact with the user, and the Awake state when the tangible coach is ready to communicate. Figure $2 \mathrm{a}$ and $\mathrm{b}$ shows respectively the sleep and awake position of the tangible coach. To switch between these two states, the user consists of touching the tangible coach and changing its position. The tangible coach aims to have a natural conversation with the user in four different languages (Italian, Spanish, Dutch, and English). The conversation can be two-sided: The user or the tangible coach can initiate the conversation.
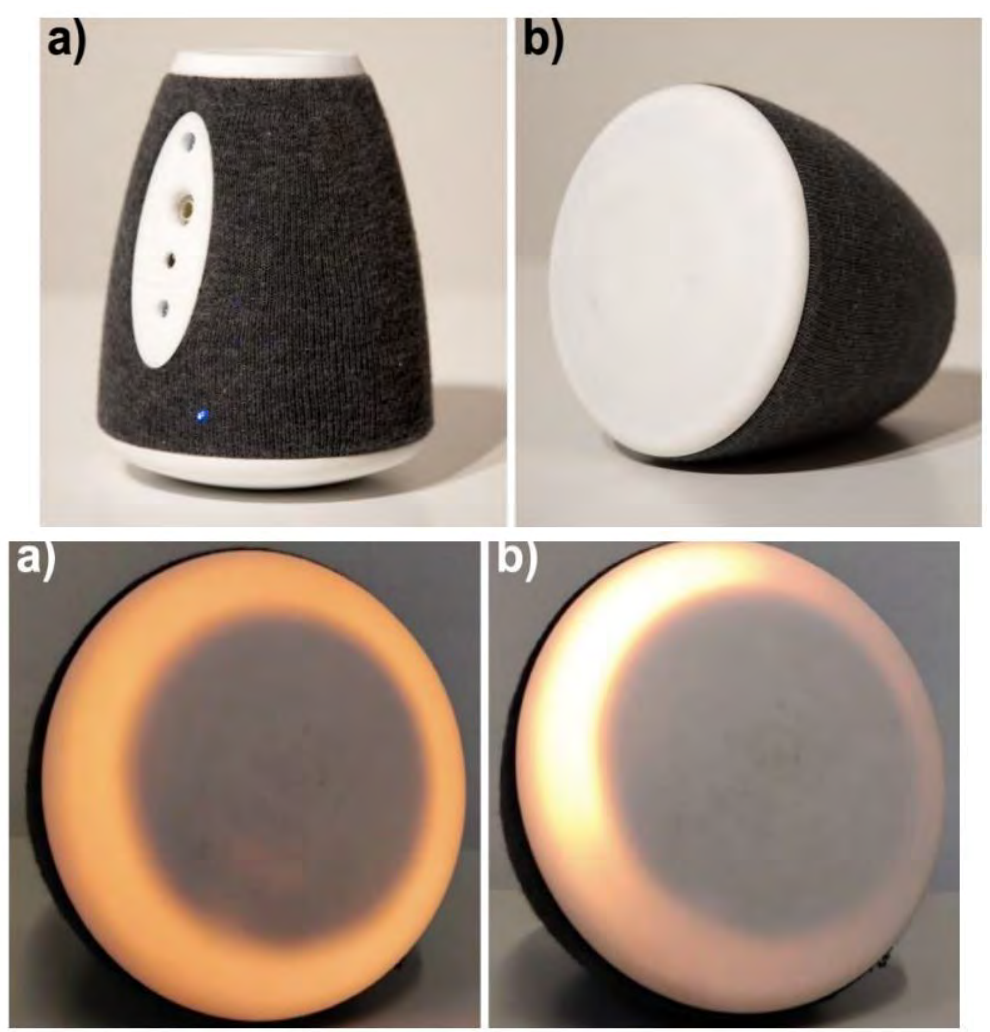

Fig. 12.5: NESTORE tangible coach final prototype

\subsection{Conclusion}


This chapter explains the co-design process method for building an embodied conversational agent. It also shows how this method was adopted in the NESTORE tangible coach project that aim to be a virtual coach for older adult' build a trustable e-coach and a companion for older adults. Vink et al[9]. explicitly linked codesign to well-being and expanded the conversation about the influence of the codesign process on well-being. The final prototype of the NESTORE tangible coach can have natural conversation with the user and follow their wellbeing score and improvement. It is currently under pilot test with 60 users in the context of the NESTORE project.

\section{References}

[1] Ullmer, B., \& Ishii, H. (2000). Emerging frameworks for tangible user interfaces. IBM systems journal, 39(3.4), 915-931

[2] Rowland, C., Goodman, E., Charlier, M., Light, A., \& Lui, A. (2015). Designing connected products: UX for the consumer internet of things. " O'Reilly Media, Inc.".

[3] Spreicer, W. (2011, June). Tangible interfaces as a chance for higher technology acceptance by the elderly. In Proceedings of the 12th International Conference on Computer Systems and Technologies (pp. 311-316). ACM.

[4] Bong, W. K., Chen, W., \& Bergland, A. (2018). Tangible User Interface for Social Interactions for the Elderly: A Review of Literature. Advances in Human-Computer Interaction, 2018.

[5] NESTORE: a companion for better and healthier ageing - Shaping Europe's digital future - European Commission https://ec.europa.eu/digital-single-market/en/news/nestorecompanion-better-and-healthier-ageing

[6] Mori, M. (1970). The uncanny valley. Energy, 7(4), 33-35.

[7] El Kamali, M., Angelini, L., Caon, M., Carrino, F., Röcke, C., Guye, S., ... \& Mugellini, E. (2020). Virtual coaches for older adults' wellbeing: A systematic review. IEEE Access, 8, 101884-101902.

[8] . Chamberlain, Paul, and Claire Craig. "Engaging design-methods for collective creativity." In International Conference on Human-Computer Interaction, pp. 22-31. Springer, Berlin, Heidelberg, 2013.

[9] Vink, J., Wetter-Edman, K., Edvardsson, B., \& Tronvoll, B. (2016, May). Understanding the influence of the co-design process on well-being. In Service Design Geographies. Proceedings of the ServDes. 2016 Conference (No. 125, pp. 390-402). Linköping University Electronic Press. 